\title{
Características físicas y alterabilidad de la piedra de Villamayor (Salamanca)
}

Dr. JORGE ORDAZ - Departamento de Petrologia y Geoquimica Facultad de Geologia - Universidad de Oviedo

\section{$R E S U M E N$}

Las propiedades físicas de la arenisca de Villamayor (Salamanca), y, en especial, la porosidad, la absorción de agua y el movimiento capilar a través de la roca, son estudiadas en relación con sus caracteristicas litológicas. Los ensayos de alterabilidad de laboratorio (humedad-sequedad, heladicidad $y$ cristalización de sales) ponen de manifiesto su susceptibilidad a los efectos destructivos de las heladas y de las fluctuaciones de humedad, y suministran datos estimativos sobre la durabilidad potencial de esta piedra de construcción.
$S U M M A R Y$

\author{
Physical properties of Villamayor sandstone \\ (Salamanca), and especially porosity, water \\ absorption and capillary flow through rock, are \\ studied in relation to its lithological characteristics. \\ Laboratory weatherability tests (wetting-drying, \\ freezing-thawing, and salt crystallisation) shows the \\ susceptibility to destructive effects of frost and \\ humidity fluctuations, and provides estimated data \\ about the potential durability of this \\ building strone.
}

\section{INTRODUCCION}

La arenisca de Villamayor (Salamanca) se ha venido utilizando, tradicionalmente, como material de construcción. Prueba de ello son los numerosos edificios y monumentos histórico-artísticos de Salamanca y provincia, en los que se ha empleado la "piedra franca de Villamayor". Su facilidad de corte y labra, aspecto agradable y patinación con el paso del tiempo son factores que han contribuido a proporcionar una especial vistosidad a este material rocoso. Sin embargo, la piedra de Villamayor, una vez puesta en obra y en contacto prolongado con los agentes de la intemperie, tiende indeclinablemente a deteriorarse bajo diversas formas de alteración (1). Esta degradación de la piedra en el monumento es consecuencia de la interacción de factores intrínsecos (características litológicas y propiedades físicas de la roca) con factores extrínsecos (agentes ambientales específicos propios del lugar de ubicación).

El objetivo principal del presente trabajo es la evaluación de la durabilidad de la arenisca de Villamayor, como material de construcción. Para ello se ha considerado la roca desde el punto de vista de su comportamiento físico, sometiendo las muestras a diversos ensayos de alterabilidad, en el laboratorio, con el fin de correlacionar los resultados obtenidos con sus características intrínsecas y aportar criterios estimativos válidos sobre su susceptibilidad y resistencia a las acciones disgregadoras del agua en sus diversas formas, en especial, frente a la del hielo.

\section{CARACTERISTICAS LITOLOGICAS}

La arenisca de Villamayor, perteneciente a los terrenos detríticos paleógenos del área salmantina, consta fundamentalmente, de cuarzo (55-70\%), feldespatos (10-15\%) y filosili- 
catos (10-15\%). Los fragmentos líticos no superan, por lo general, el $10 \%$. Dicha composición mineralógica la acerca a las areniscas de tipo arcósico. Los feldespatos son mayoritariamente potásicos (ortosa y microclima) y, en menor medida, plagioclasas. Más de la mitad de los filosilicatos (que forman parte del cemento de la roca) son illitas e interestratificados illita-montmorillonita. En menor proporción se encuentra clorita, caolinita y montmorillonita. El porcentaje de minerales arcillosos de carácter expansivo puede cifrarse en un 5-8 \%. Entre los principales minerales accesorios $(\simeq 1 \%)$ se encuentran: moscovita, granate, epidota, andalucita, turmalina y opacos. También existen pequeñas cantidades de óxidos e hidróxidos de hierro, de carácter secundario.

El tamạño de grano medio es algo variable, y se sitúa, para las muestras estudiadas, alrededor de 0,2 mm (tamaño arena fina). En general están bien calibradas, y las curvas de distribución granulométricas son simétricas. La fábrica no evidencia orientaciones granulares preferentes. Los contactos entre los granos son, normalmente, de tipo tangente y largo. Al microscopio óptico y, sobre todo, al electrónico de barrido (SEM) se pueden observar perfectamente los numerosos espacios huecos (poros), de carácter intergranular o intersticial, así como las formas de los filosilicatos cementantes (especialmente illitas), de hábitos cristalográficos filamentosos en agregados muy desarrollados, figs. 1 y 2.

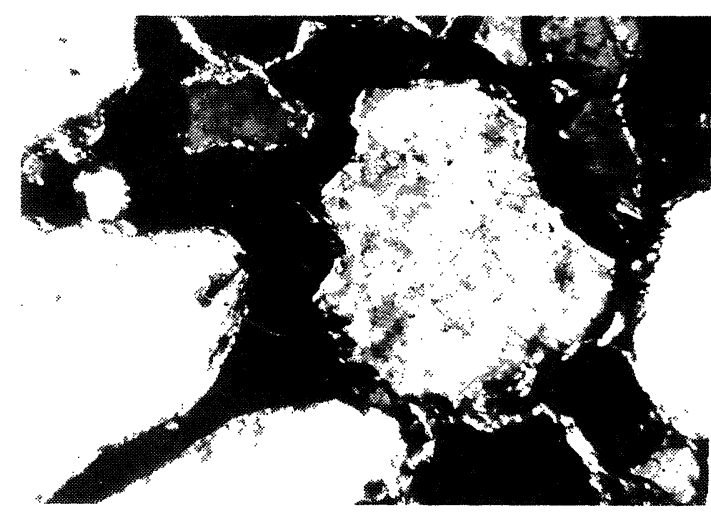

Fig. 1.-Aspecto de la textura de la arenisca de Villamayor. Obsérvense la abundancia de espacios porcsos (en negro), y los recubrimientos de filosilicatos alrededor de los granos minerales. N.C. $\times 200$.

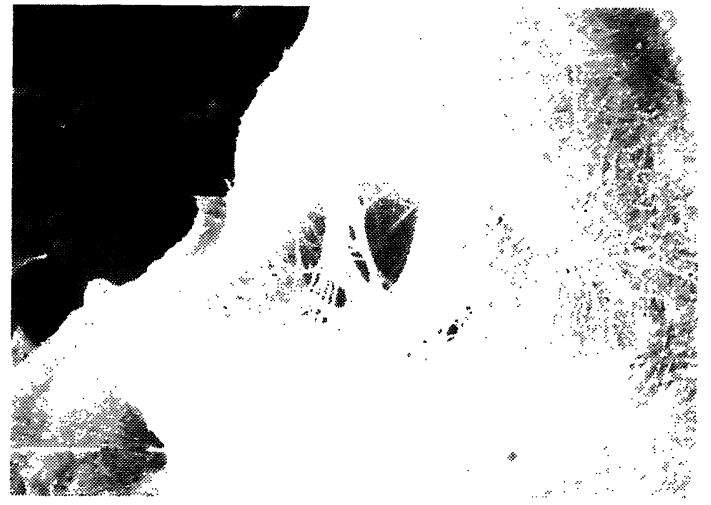

Fig. 2.-Detalle de la superficie de un grano tapizada por un agregado filamentoso de illita, y de los conductos porosos. (SEM, 1 división $=10$ $\mu \mathrm{m})$.

\section{PROPIEDADES FISICAS}

Al objeto de caracterizar físicamente la arenisca de Villamayor, se determinaron varios parámetros físicos, en especial los relacionados con la sorción y movimiento de fluidos (agua) por el interior de la roca, y que, en principio tienen, por sus derivaciones, mayor implicación en los fenómenos de deterioración de dicho material rocoso.

Para ello se seleccionaron varios bloques de roca extraídos de las canteras en explotación "Sanchón" y "Arrecias", ubicadas en el térmmino municipal de Villamayor, y representativos de la llamada "piedra de Villamayor" de calidad, empleada en la construcción y restauración de monumentos. De estos bloques se extrajeron, asimismo, las muestras utilizadas en los estudios petrográficos. Para la medición de las propiedades físicas se tuvieron en cuenta, básicamente, las recomendaciones propuestas por la ISRM, excepto para los ensayos de capilaridad, en los que se siguió el denominado "ensayo francés", descrito, entre otros, por Mamillan (2). Los resultados obtenidos, para las distintas propiedades, se muestran en la Tabla I. 


\begin{tabular}{ll} 
& \multicolumn{1}{c}{ Promedio } \\
\cline { 2 - 2 } Densidad de los granos minerales $\left(\rho_{\mathrm{s}}\right)$ & $2,66 \mathrm{~g} / \mathrm{cm}^{3}$ \\
Densidad aparente de la roca seca $\left(\rho_{\mathrm{d}}\right)$ & $1,76 \mathrm{~g} / \mathrm{cm}^{3}$ \\
Porosidad abierta $\left(n_{\mathrm{o}}\right)$ & $32,9 \%$ \\
Porosidad total $(n)$ & $33,8 \%$ \\
Grado de saturación $\left(S_{\mathrm{r}}\right)$ & $95,9 \%$ \\
Contenido en humedad natural $(w)$ & $17,0 \%$ \\
Absorción de agua $(W)$ & $14,5 \%$ \\
Contenido en humedad de saturación $\left(i_{\mathrm{s}}\right)$ & $18,4 \%$ \\
Coeficiente de hinchamiento $\left(\varepsilon_{\mathrm{s}}\right)$ & $2,55 \times 10^{-3}$ \\
Coeficiente de capilaridad $(C)$ & $120 \mathrm{~kg} / \mathrm{m}^{2} \cdot \mathrm{min}^{0,5}$ \\
Coeficiente de penetración de agua $(A)$ & $5,6 \times 10^{-3 \cdot} \mathrm{m} / \mathrm{min}^{0,5}$ \\
Coeficiente de expansión térmica $\left(\alpha_{1}\right)$ & $9,0 \mathrm{~m} / \mathrm{m}^{\circ} \mathrm{C}$
\end{tabular}

\section{COMENTARIOS A LAS CARACTERISTICAS FISICAS}

En primer lugar se trata de un material de elevada porosidad, aunque algo variable $\left(n_{0}\right.$ entre 28-35\%). Los espacios porosos representan, pues, alrededor de un tercio del volumen de la roca. La gran diferencia observada entre $\rho_{\mathrm{g}}$ y $\rho_{\mathrm{d}}$ nos confirma la abundancia de huecos. Además, la porosidad efectiva no se halla muy cercana a la $n$, siendo el $S_{\mathrm{r}}$ muy alto; lo que significa que la mayor parte de los poros están intercomunicados. La conectividad de los canales porosos que unen los poros entre sí, tiene como resultado inmediato la facilitación del flujo de fluidos por el interior de la roca. La higrospicidad, o capacidad para absorber agua de esta arenisca es igualmente alta, como se desprende de los valores de $W$ e $i_{\mathrm{s}}$. El "coeficiente de saturación" se sitúa aproximadamente en un $40 \%$. La absorción de agua - tanto en fase líquida como en fase de vapor (humedad ambiental) se efectúa preferentemente en las primeras horas del ensayo: en 24 horas el agua absorbida supera el $75 \%$ del total. Del mismo modo, la mayor parte $(>70 \%)$ de la desorción en muestras saturadas de agua, por desecación (evaporación) en condiciones ambientales, tiene lugar igualmente en un tiempo similar. Se puede decir, por tanto, que la transferencia de fluidos a través del entramado de poros intercomunicados, tanto hacia dentro como hacia fuera de la roca, se realiza con relativa celeridad.

En el terreno, y debido a la proximidad de las aguas subterráneas (freáticas) y superficiales (fluviales), la roca - sobre todo la de los lechos más cercanos al acuífero- se presenta con un grado de humedad natural variable pero alto, próximo a su $i_{\mathrm{s}}$. Este elevado contenido en agua hace que la resistencia mecánica (en especial a la tracción) de la roca se vea considerablemente disminuida, partiéndose los bloques con suma facilidad, con lo que el corte y la labra de los mismos, en estas condiciones, resulta extraordinariamente cómodo. Fuera de su entorno propio, sin embargo, y por progresiva evaporación del agua encerrada en sus poros al ser expuesta a las condiciones de temperatura y humedad ambientales, la roca va perdiendo su "agua de cantera", con relativa rapidez, al tiempo que se contrae ligeramente y endurece paulatinamente con el tiempo. La retracción por desecación, insignificante a pequeña escala, puede, sin embargo, ser manifiesta en los grandes bloques o lascas al suponer un acortamiento lineal, medido experimentalmente, de alrededor del $0,3 \%$, lo que viene a representar unos $3 \mathrm{~mm}$ por metro. fig. 3 . La contracción puede ser aún mayor en el caso de que la piedra pierda totalmente su humedad natural. 
En este caso - prácticamente inalcanzable en condiciones naturales- el acortamiento puede ser del orden de unos $8 \mathrm{~mm}$ por metro, o incluso más. En cualquier caso, la disminución dimensional va generalmente acompañada de un incremento relativo de la resistencia mecánica y dureza de la roca, que se traduce, en la práctica, en un aumento de las dificultades a ser trabajada. Es de señalar que, a pesar de que la piedra "curada" sea posteriormente vuelta a saturar en agua, no se recobra de una manera total su "blandura" inicial, ni tampoco se recuperan totalmente las dimensiones originales del bloque antes de la retracción.

No obstante, cuando la roca se humedece o empapa en agua, sufre una expansión volumétrica no muy elevada, pero sí significativa, fig. 4. Este hinchamento es debido, esencialmente, a la presencia de minerales arcillosos considerados expansivos (montmorillonita e interestratificados illita-montmorillonita, principalmente), con lo que, en principio, aquellas muestras de arenisca que contengan una mayor proporción de estos filosilicatos, serán también las que, proporcionalmente, exhiban mayores hinchamientos. Sin embargo es arriesgado presuponer una proporcionalidad lineal entre el contenido de las arcillas expansivas y el coeficiente de hinchamiento, dado que otros factores de carácter textual interfieren generalmente en el resultado final de la expansión. Así, a igualdad de contenido en filosilicatos hinchables, las muestras más porosas tienden a presentar, casi siempre, $\varepsilon_{\mathrm{s}}$ proporcionalmente mayores que las menos porosas. En consecuencia, la fábrica de la arenisca, centrada en el empaquetamiento y ordenación espacial de los granos minerales, y la distribución e interconexión de los espacios vacíos, serían rasgos texturales que parcialmente controlarían y determinarían las cualidades y cuantía del hinchamiento final.

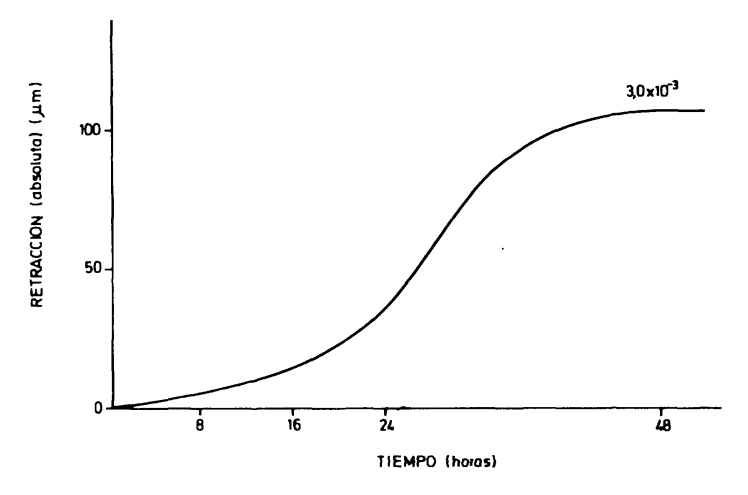

Fig. 3.-Ejemplo de curva de retracción de una muestra de arenisca de Villamayor con humedad natural, por desecación en condiciones ambientales $\left(\right.$ Tem. $=15-20^{\circ} \mathrm{C} ;$ H.r. $\left.=70-80 \%\right)$.

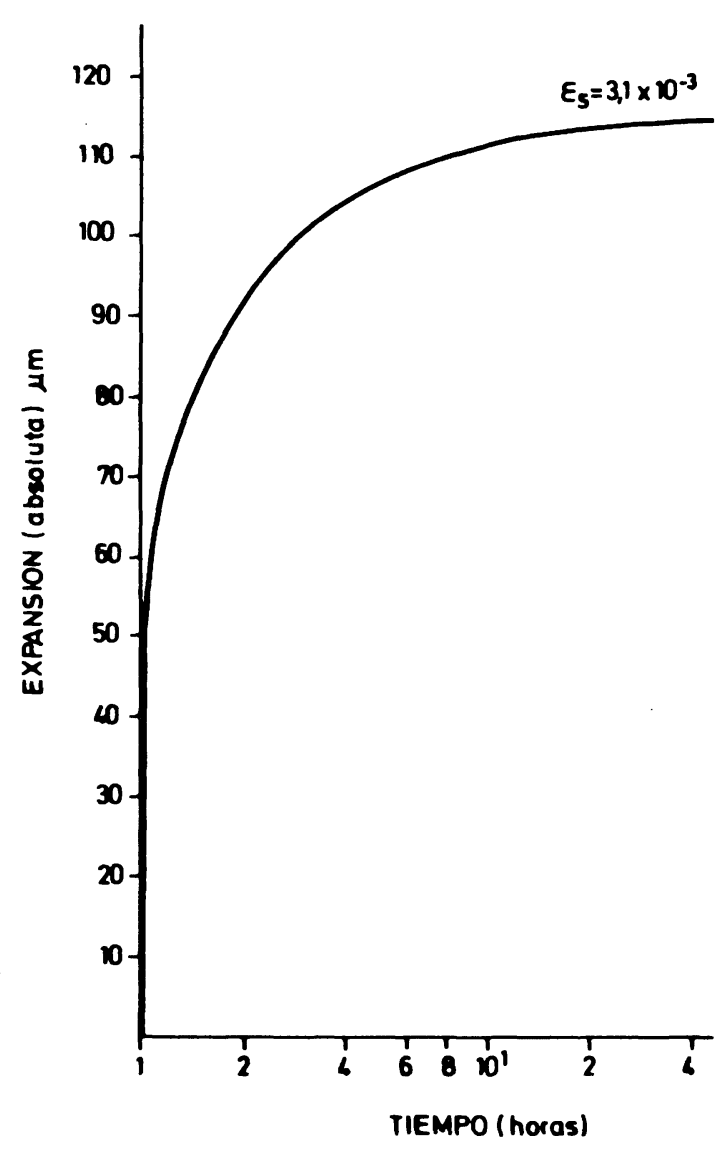

Fig. 4.-Curva típica de expansión, por imbibición en agua, con respecto al tiempo $\left(\varepsilon_{\mathrm{s}}=\right.$ coeficiente de hinchamiento). 
Por otro lado, las medidas de capilaridad ponen de relieve la habilidad, para la succión capilar de agua, de la piedra de Villamayor. Los relativamente altos valores de $\ddot{A}$ y de $C$, caracterizan dicha propiedad, fig. 5. La altura alcanzada por la ascensión capilar del agua está en función, esencialmente, del tamaño y forma de los poros y canales porosos: cuanto más pequeños sean, mayor será también la fuerza (presión) capilar y, consecuentemente, la altura lograda por el agua en su recorrido ascendente; toda vez que dicha subida por los capilares de la roca es inversamente proporcional al diámetro de los mismos. El porcentaje de microporos (radio de entrada $<7 \mu \mathrm{m}$ ) juega, por tanto, un papel decisivo en el régimen de capilaridad. En el caso de la arenisca de Villamayor la abundancia de microporos es variable según las muestras. fluctuando los porcentajes de microporosidad, con respecto a la porosidad abierta entre un $50 \%$ y un $95 \%$. Dichos porcentajes, así como los valores de distribución de tamaños de los conductos de acceso a los poros, se obtuvieron a partir de ensayos porosimétricos por inyección de mercurio (3). Es evidente, pues, que aquellas variedades que presenten una mayor cantidad de microporos intercomunicados, para una $n_{0}$ dada, serán las que, en principio, evidenciarán una mayor capacidad de sección de agua por capilaridad, como así se ha podido constatar.

Respecto a la expansión térmica, los valores registrados de $\alpha_{1}$ están en función, primariamente, del contenido en cuarzo, que es, entre los minerales esenciales de la roca, el que mayor dilatación térmica individual exhibe, fig. 6. Las características texturales (especialmente del sistema poroso), también influyen en los valores obtenidos, sobre el supuesto de que, a igualdad de otros parámetros petrográficos, cuanto menores sean los vacíos en el material rocoso, mayor será, en principio, el efecto dilatacional último.

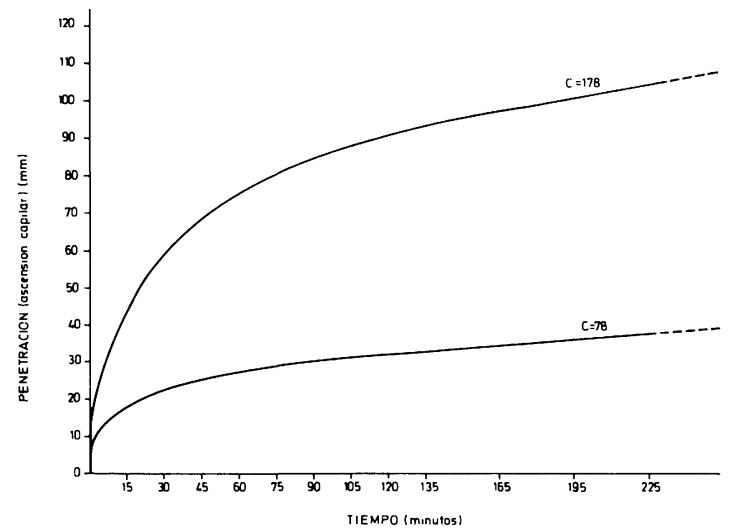

Fig. 5.-Dos ejemplos de curvas de ascensión capilar de agua, con respecto al tiempo, en las primeras horas de ensayo. ( $\mathbf{C}=$ coeficiente de capilaridad).

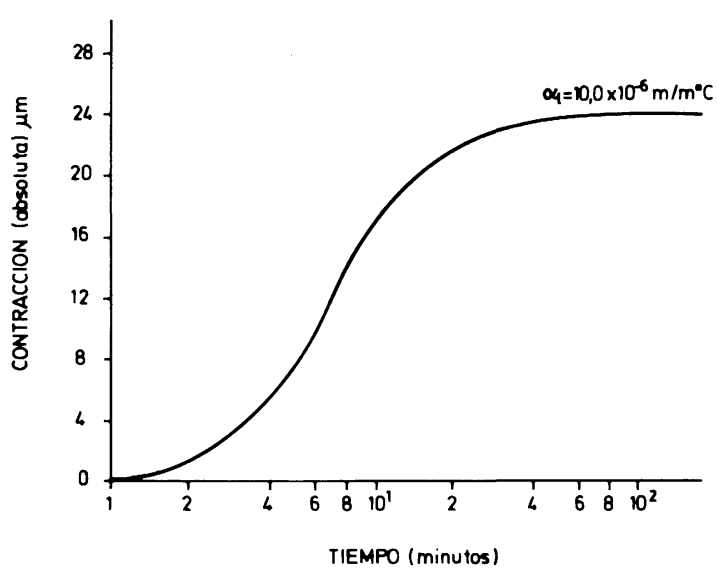

Fig. 6.-Curva típica de contracción térmica, con respecto al tiempo. (Intervalo de temperaturas de $60^{\circ} \mathrm{C}$; temp. máxima de $80^{\circ} \mathrm{C} ; \alpha_{1}=$ coeficiente de expansión térmica).

\section{ALTERABILIDAD}

Se puede definir la alterabilidad de un material rocoso como la tendencia a alterarse o meteorizarse a corto plazo (escala histórica), generalmente fuera de su entorno geológico natural (p. ej. una vez colocado en un edificio o monumento). El contenido de la alterabilidad o meteorabilidad de una roca es de primordial importancia para entender su comportamiento frente a los agentes deteriorantes externos. La durabilidad, inversamente a la alterabilidad, viene definida como la resistencia de la roca al debilitamiento y degradación cuando es sometida a procesos de alteración a corto plazo (4).

Diversos "ensayos de alterabilidad" se han ideado conducentes a la caracterización física 
de las rocas en relación a aquellos factores que de una manera más determinante coadyuvan a la alteración de las mismas. En este aspecto, el agua (en todas sus formas) es uno de los factores más agresivos. Los ensayos efectuados en este trabajo giran, pues, alrededor de la susceptibilidad de la arenisca de Villamayor a la acción del agua.

\section{Ciclos de humedad-sequedad}

Los ensayos basados en sucesivos ciclos alternos de imbibición en agua (humedad) y evaporación (sequedad) suministran datos de interés relativos a la tendencia a la degradación de la piedra por fluctuaciones del contenido en humedad. De entre los varios tipos de ensayos propuestos se ha seguido, en nuestro caso, el siguiente procedimiento:

1) Inmersión en agua de las muestras (en número de $5 \mathrm{y}$ de $25-45 \mathrm{~g}$ de peso), durante 24 horas.

2) Secado de las muestras a estufa $\left(105^{\circ}-110^{\circ} \mathrm{C}\right)$, durante 12 horas.

El número de ciclos realizados fue de 30 . Después de cada cinco ciclos se pesó en seco el material disgregado de cada muestra. Al término del ensayo se anotó el total acumulado, expresándose en forma de porcentaje con respecto al peso inicial en seco de la muestra. Las pérdidas de material por desagregación contabilizadas al final de los 30 ciclos fue variable para las muestras ensayadas, oscilando entre el $0,8 \%$ y el $6,4 \%$. El promedio fue de $3,2 \%$. Los resultados obtenidos, así como el de los otros ensayos, se muestran en la Tabla II.

TAB L A II

Pérdida de material por desagregación en los ensayos de alterabilidad

\begin{tabular}{|c|c|c|c|}
\hline Ensayo & N. Ciclos $^{-}$ & N. ${ }^{\circ}$ Muestras & $\begin{array}{l}\text { Porcentajes de pérdida de } \\
\text { material (en peso) }\end{array}$ \\
\hline Humedad-sequedad & 30 . & 5 & $0,8-6,4 \%$ (prom. $3,2 \%$ ) \\
\hline Hielo-deshielo & 30 & 5 & $3,0-9,5 \%$ (prom. $5,7 \%$ ) \\
\hline Cristalización de sales & 10 & 5 & $15,1-29,7 \%$ (prom. $20,8 \%$ ) \\
\hline
\end{tabular}

\section{Ciclos de hielo-deshielo (heladicidad)}

El hielo ejerce una acción destructiva muy notable en las piedras debido, particularmente. al incremento de volumen al pasar el agua líquida contenida en poros y fisuras, al estado sólido, lo que genera esfuerzos internos de tracción que en última instancia y tras repetidos ciclos, pueden deteriorarlas gravemente llegando, en ocasiones, por fatiga, a su desmoronamiento o ruptura total (gelifracción). Los ensayos de heladicidad o gelivación consisten básicamente en someter una serie de muestras de roca a sucesivos ciclos de congelación y descongelación (hielo-deshielo), con objeto de evaluar la resistencia a las heladas de dicha roca. En nuestro caso se utilizaron probetas cúbicas de unos 3-5 cm de arista. Antes del ensayo las muestras fueron secadas y pesadas, y sus dimensiones medidas. Las aristas y vértices fueron pintados con tinta indeleble a fin de hacer más visibles los deterioros superficiales en las probetas durante el experimento. El procedimiento seguido fue el siguiente:

Las probetas se sumergieron en agua durante una semana, tiempo considerado como suficiente para asegurar una buena imbibición. A continuación se introdujeron en una cáma- 
ra climática cuya temperatura se encontraba entre $5^{\circ}$ y $10^{\circ} \mathrm{C}$. A partir de este momento la temperatura de la cámara se hizo descender gradualmente hasta $-3^{\circ} \mathrm{C}$, manteniéndose en este punto dos horas. Luego la temperatura se volvió a bajar hasta $-14^{\circ} \mathrm{C}$, en donde se mantuvo por espacio de cuatro horas. La velocidad de enfriamiento fue de $0,24^{\circ} \mathrm{C} / \mathrm{min}$., es decir, aproximadamente un grado centígrado cada cuatro minutos.

Terminada la fase de congelación, las probetas fueron desheladas introduciéndolas en un recipiente con agua a temperatura ambiente, durante un tiempo de dos horas como mínimo. Acabada esta fase de descongelación, las probetas fueron de nuevo colocadas en la cámara frigorífica para empezar otro ciclo idéntico. El número total de ciclos efectuados fue de 30. Los cambios sufridos por las muestras tras cada ciclo (efectos superficiales, desprendimiento de granos o fragmentos, aparición de fisuras, etc.) fueron debidamente anotados. Una vez terminados los ciclos de hielo-deshielo, las probetas fueron pesadas, en seco, para conocer la pérdida de material por desagregación. También se midieron de nuevo sus dimensiones con el calibre.

Ninguna de las cuatro probetas ensayadas se fracturó o se desmoronó completamente durante el ensayo; aunque una de ellas sufrió serios daños, sobre todo alrededor de los vértices. Durante los primeros diez ciclos sólo se observaron pequeños desprendimientos de granos, en la fase de inmersión en agua (descongelación). A partir del décimo ciclo se empezaron a notar ya ligeros redondeamientos en los vértices, así como pequeños desconchones a lo largo de las aristas. Las superficies se hicieron más ásperas, con desprendimiento de granos (arenización) por simple contacto con los dedos. A partir del vigésimo ciclo, las descamaciones, desconchaduras y fisuraciones se hicieron más generales y manifiestas. La pérdida media de material por desagregación al cabo de los treinta ciclos de hielodeshielo fue de $5,7 \%$.

\section{Cristalización de sales}

Los ensayos de "cristalización de sales" tienen por objeto evaluar el comportamiento físico de una roca frente a la acción disruptiva de soluciones salinas al cristalizar en el interior de poros. Dichos ensayos se basan, esencialmente, en repetidos ciclos de inmersión de las muestras rocosas en soluciones salinas más o menos concentradas, y posterior desecación; determinándose al final de los ciclos las posibles variaciones y deterioros que hayan podido sufrir las muestras desde el punto de vista de sus características físicas. Con ello se obtiene, como en otros ensayos de alterabilidad similares, una aproximación de su durabilidad frente a agentes exógenos agresivos.

Por otro lado, aceptada la "intensificación" o "aceleración" que comporta este tipo de pruebas experimentales, en comparación con las condiciones naturales, dichos ensayos pueden también considerarse como de "envejecimiento artificial" de la roca, indicadores de su proclividad a la disgregación física bajo los efectos de la intemperie, especialmente de la acción de las heladas.

Para la realización de este ensayo se ha seguido, básicamente la norma DIN 52111, pero con algunas variantes procedimentales propuestas por autores como Marschner (5) y Price (6). Se utilizaron cubos de piedra de $3-4 \mathrm{~cm}$ de arista, previamente secados a estufa. La solución salina empleada fue una solución acuosa de $\mathrm{SO}_{4} \mathrm{Na}_{2}$ al $15 \%$. El efecto disruptivo del $\mathrm{SO}_{4} \mathrm{Na}_{2}$, es debido al incremento de volumen de un $400 \%$ durante su transición a $\mathrm{SO}_{4} \mathrm{Na}_{2} \cdot 10 \mathrm{H}_{2} \mathrm{O}$, en una solución acuosa a temperatura inferior a $32,4^{\circ} \mathrm{C}$.

Las probetas fueron sumergidas parcialmente en la solución por espacio de 5 horas, a una temperatura de unos $18^{\prime \prime} \mathrm{C}$. Posteriormente las probetas fueron secadas a estufa (precalen- 
tada a unos $70^{\circ} \mathrm{C}$ ) durante 15 horas, como mínimo, a $105-110^{\circ} \mathrm{C}$. Luego se dejaron enfriar a temperatura ambiente, dentro de un desecador, aproximadamente durante una hora, al cabo de la cual volvieron a ser sumergidas en la solución del sulfato sódico. El número de ciclos efectuados fue de 10.

Terminados los ciclos, las probetas, después de ser dejadas en agua durante 12 horas como mínimo para remover las sales, fueron pesadas en seco, y su peso comparado con el inicial, a fin de conocer el porcentaje de pérdida de material, que en nuestro caso fue del $20,8 \%$, en promedio. Todas las muestras ensayadas resistieron los 10 ciclos sin desintegrarse o desmoronarse totalmente, aunque evidenciaron una apreciable reducción en su tamaño debido a la pérdida de material granular por desagregación, a medida que se avanzaba en los ciclos.

\section{VALORACION DE LOS RESULTADOS}

La finalidad principal de los ensayos de la alterabilidad realizados es, como se ha dicho, la de suministrar datos que permitan la evaluación de la resistencia a la alteración física (mecánica) a corto plazo, por parte de la piedra de Villamayor; es decir, de su durabilidad potencial.

En este sentido, los resultados obtenidos en los diferentes ensayos nos indican que el agua - tanto en forma líquida como en forma de hielo- ejerce una acción destructiva decisiva de cara a la durabilidad de este material pétreo. La acción disgregadora del agua se manifiesta, principalmente, en todos los ensayos realizados, a través de la pérdida progresiva del material superficial por desagregación manteniendo las muestras, en todo caso, una cierta coherencia interna o cohesión intergranular, sin llegar al menos en el número de ciclos a las que fueron sometidas a resquebrajamientos o fracturaciones generalizadas.

La pérdida de material fue significativa en el ensayo de heladicidad, en donde los efectos crioclásticos del hielo resultaron ser proporcionalmente más resolutivos que los efectos derivados de las meras fluctuaciones del nivel hídrico de las muestras (constatados a partir de los ensayos de humedad-sequedad), para el mismo número de ciclos. El ensayo de cristalización de sales debe contemplarse, a efectos evaluativos, como una magnificación o aceleración del efecto de disrupción del hielo; de especial significación en el caso particular de las piedras de los monumentos que contengan, de añadidura, previsibles cantidades de determinadas sales solubles. En este sentido, Dunn y Hudec (7), asignan, para una solución del $10 \%$ de $\mathrm{SO}_{4} \mathrm{Na}_{2}$, un factor de deterioro de más de diez, en relación con el daño producido por un número equivalente de ciclos de hielo-deshielo.

Normalmente el desprendimiento granular va acompañado también de empobrecimiento de partículas de la fracción arcillosa cementante (descementación) que, a su vez, favorece ulteriores separaciones de los granos, ya de por sí pobremente trabados. En cambio, el deterioro físico de las muestras ensayadas parece no repercutir severamente sobre su capacidad de absorción de agua. Las mediciones de $W$ efectuadas en este sentido antes y después de los ensayos de alterabilidad, no muestran desviaciones significativas, pudiéndose afirmar que permanecen aproximadamente dentro del rango de valores inicial de las muestras no ensayadas, y sin que se pueda establecer, en líneas generales, ninguna tendencia clara a aumentar o a disminuir. Sólo la aparición ocasional de algunas fisuras generadas durante los ensayos - por otro lado no muy abundantes - pueden llegar a incrementar sensiblemente el volumen de espacios vacíos de la roca y, en consecuencia, aumentar la absorción rápida de agua. 
Se ha visto en los ensayos realizados que, por lo general, las muestras que exhiben mayores pérdidas de material y daños físicos corresponden, en principio, a las que contienen mayores porcentajes de filosilicatos (especialmente illita e interestratificados). Por consiguiente, parece lógico pensar que la mayor o menor cantidad de cemento arcilloso, y la naturaleza del mismo, son factores determinantes a la hora de calibrar, en nuestro caso, la contribución parcial de los componentes mineralógicos a la alterabilidad de la arenisca de Villamayor; sin perjuicio de otros factores texturales coadyuvantes, como puedan ser, geometría del sistema poroso, el grado de conectividad entre los poros, el tamaño de grano y las uniones intergranulares, etc.

La distribución del tamaño de los poros es, a este respecto, otro de los factores condicionantes más relevantes, en la medida en que, cuanto menor es el diámetro de entrada de los poros, mayores son las presiones de cristalización que transmiten el hielo o las sales a las paredes de los poros, provocando tensiones internas, y posibilitando, de esta forma, la apertura de nuevas vías (microfisuras) de acceso a los fluidos (particularmente agua) hacia el interior de la piedra y viceversa. En efecto, Walker et al. (8) estableccieron un tamaño crítico de poro, de aproximadamente $5 \mu \mathrm{m}$, en vistas a la resistencia a la gelifracción de los materiales porosos. Diámetros medios superiores al citado tamaño permitirían un fácil drenaje y expulsión hacia fuera de fluidos del avance frontal de la línea de congelación, con lo que estos poros contribuirían a aminorar, en parte, los efectos disruptivos del hielo.

En la arenisca de Villamayor, el espectro de tamaños de los poros es bastante amplio y variable. En las figs. 7 y 8 , se muestran dos histogramas distintos de distribución de los radios de acceso de los poros, obtenidos a partir de los ensayos porosimétricos (3). Como puede observarse, existen marcadas diferencias entre ambas representaciones. La muestra de tipo A presenta la mitad de su porosidad concentrada en el intervalo de 6-20 $\mu \mathrm{m}$; mientras la otra mitad aparece homogéneamente repartida dentro de una amplia gama de $0,004 \mu \mathrm{m}$ a $6 \mu \mathrm{m}$. La muestra de tipo B presenta, por lo contrario, una repartición más uniforme, con la moda alrededor de $0,3 \mu \mathrm{m}$, siendo significativo el intervalo de $0,01 \mu \mathrm{m}$ a $4 \mu \mathrm{m}$, característico del tamaño de microporo, e inferior, en su mayor parte, al citado radio crítico de $2,5 \mu \mathrm{m}$.
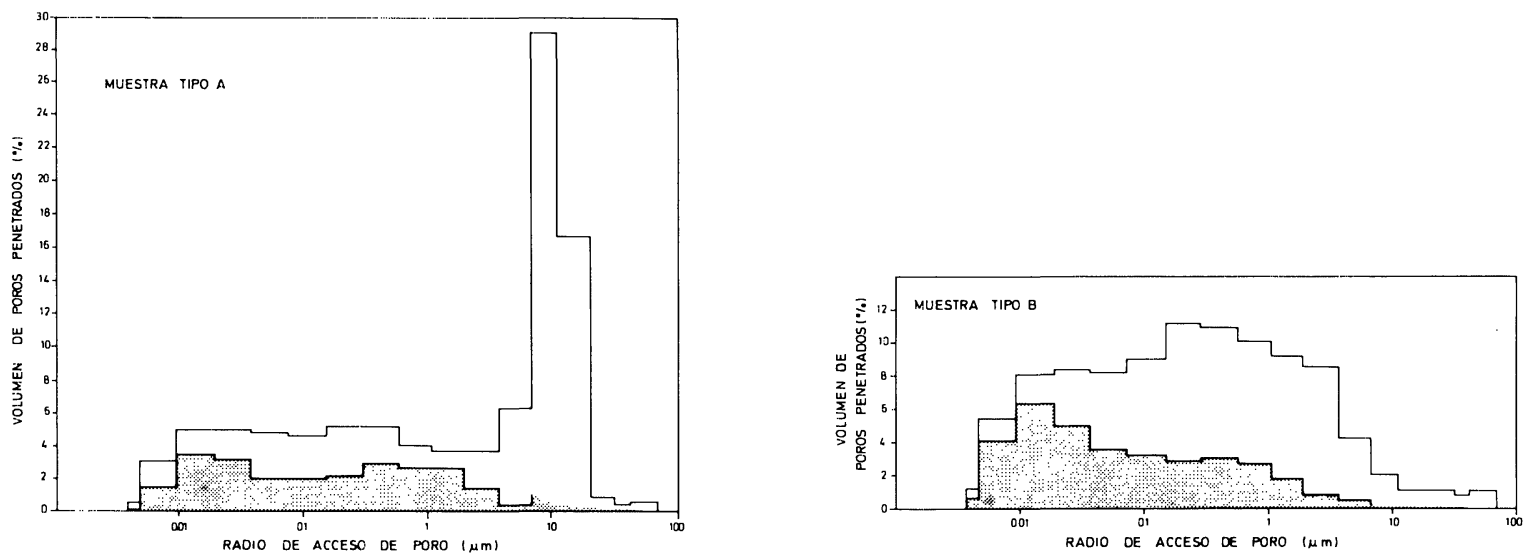

Figs. 7 y 8.-Histogramas de variación del volumen de poros penetrados por mercurio con el radio de acceso de los poros, para dos muestras de arenisca de Villamayor (tipos A y B). El volumen de poros "atrapados" en cada intervalo de radios de acceso de poros, aparece sin puntear.

En los ensayos de heladicidad y cristalización de sales se pudo comprobar que las mayores pérdidas relativas de material granular correspondían a aquellas muestras similares al 
tipo B; es decir, con un mayor porcentaje de microporosidad. A ello se sumaría el efecto añadido de la especial morfología de estos microporos, con canales de entrada muy estrechos, de cuello de botella, o tortuosos, lo que hace que, una vez dentro, el líquido penetrante quede parcialmente "atrapado", sin poder volver a salir de aquellos. Esta "porosidad atrapada" $n$ (atp) (que se muestra, también, en los histogramas) se deduce a partir de los datos obtenidos en dos inyecciones consecutivas de mercurio en una misma probeta. Como puede observarse en las figs. 7 y 8 , la $n$ (atp) corresponde, principalmente, al segmento de macroporosidad, en ambos tipos de muestras. Las de tipo B, sin embargo, tendrían, además, gran parte de su microporosidad atrapada, mientras que en las de tipo A sería mucho menor. Esta particular porosidad ejercería, de hecho, una influencia notable en la retención de humedad por parte del material rocoso $\mathrm{y}$, por ende, en la acción disgregadora del agua o hielo, sobre todo en aquellas muestras cuya microporosidad tuviese en su mayoría un carácter atrapado. Este es el caso de las muestras de arenisca de tipo B cuyos microporos de radio inferior a 2,5 $\mu \mathrm{m}$ (hasta aproximadamente $0,05 \mu \mathrm{m}$ ) son mayoritariamente atrapados, por lo que las posibilidades de que se vean negativamente afectadas por las acciones disruptivas anteriormente mencionadas serían mayores, dsminuyendo con ello su durabilidad potencial.

\section{CONCLUSIONES}

De las observaciones realizadas y resultados obtenidos en los distintos ensayos, se pueden entresacar las siguientes conclusiones:

1. La arenisca de Villamayor se caracteriza desde el punto de vista físico, entre otras cualidades, por una elevada porosidad abierta (en gran medida constituída por microporos), facilitadora de la absorción de agua y del flujo capilar a través de la misma.

2. Los ensayos de alterabilidad efectuados (humedad-sequedad, heladicidad y cristalización de sales) ponen en evidencia la susceptibilidad de la piedra de Villamayor a las fluctuaciones del contenido en humedad y, en especial, a la acción disruptiva del hielo; manifestada, principalmente, a través de la pérdida progresiva de material superficial por desagregación y descementación.

3. La presencia y naturaleza de los filosilicatos que constituyen el cemento de la arenisca, así como el espectro de tamaños de los poros, son, en principio, los factores mineralógicos-texturales de mayor influencia respecto a la durabilidad de este material rocoso. En este sentido, se ha constatado que, en líneas generales, aquellas variedades litológicas de piedra de Villamayor que contienen mayores cantidades de minerales arcillosos expansivos y mayor porcentaje de microporosidad, son las que, en principio, se ven más afectadas por las acciones disgregadoras del agua en sus diversas formas.

\section{AGRADECIMIENTOS}

El presente trabajo se ha realizado gracias a una Ayuda a la Investigación de la Caja de Ahorros y Monte de Piedad de Salamanca (Convocatoria de 1981).

\section{BIBLIOGRAFIA}

(1) Ordaz, J. (1982): Estado de alteración y alterabilidad futura de la piedra de Villamayor de los monumentos salmantinos: interpretación petrográfica. (Memoria inédita), Departamento de Petrologia y Geoquimica, Universidad de Oviedo, $111 \mathrm{p}$.

(2) Mamilan, M. (1981): Connaissances actuelles des problèmes de remontées d'eau par capillarité dans les murs, The Conservation of Stone, pp. 59-77. 
(3) Ordaz, J. y Alonso, F. J. (1983): Caracteristicas del sistema poroso de la arenisca de Villamayor (Salamanca), Trabajos de Geología, Univ. de Oviedo, (in litt).

(4) Fookes, P. G.; Dearman, W. R. y Franklin, J. A. (1971): Some engineering aspects of rock weathering with field examples from Dartmoor and elsewhere, Q. Jl. Engng. Geol., 4, pp. $139-185$.

(5) MARSChNeR, H. (1978): Application of salt crystallisation test to impregnated stones, Proc. Int. Symp. on Deterioration and Protection of Stone Monuments, París, T-I, 3.4, $16 \mathrm{p}$.

(6) PrICE, C. A. (1978): The use of sodium sulphatecrystallisation test for determining the weathering resistance of untreated stone, Proc. Int. Symp. on Deterioration and Protection of Stone Monuments, Paris, T-I, 3.6, 23 p.

(7) Dunn, J. R. y Hudec, P. P. (1966): Water, clay and rock soundness, Ohio Journal of Science, 66 (2), pp. 153-167.

(8) Walker, R. D.; Pence, H. J.; Hazlet, W. H. y ONG, W. J. (1969): One-cycle slow-freeze test for evaluation aggregate performance in frozen concrete, National Cooperative Highy Research Program Report, 65, $21 \mathrm{p}$.

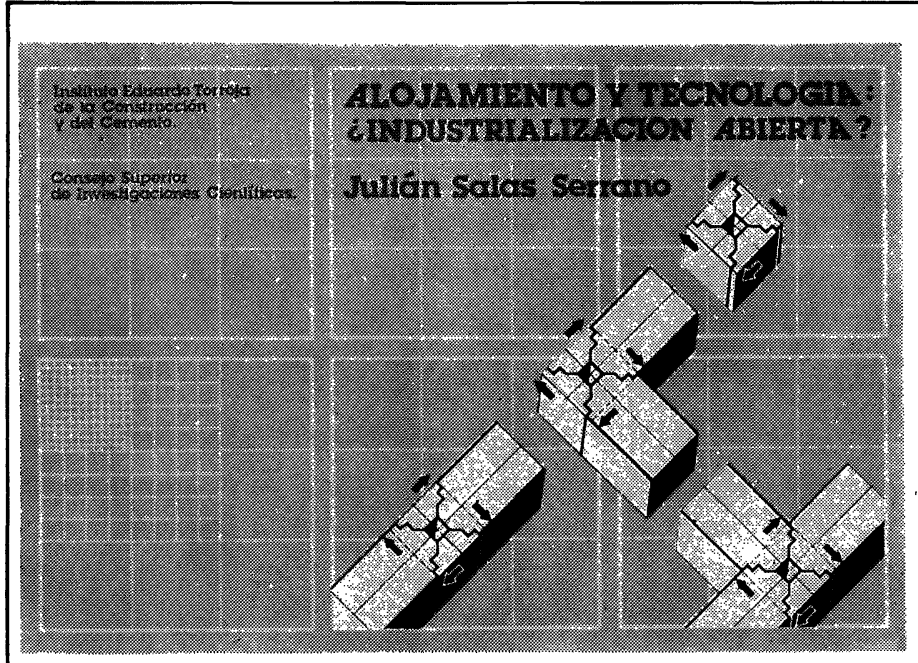

\section{ALOJAMIENTO Y TECNOLOGIA:} ¿INDUSTRIALIZACION ABIERTA?

JULIAN SALAS, ING. IND. (I.E.T.c.c.)

Un volumen de 160 páginas, 109 figuras y 16 tablas. Tamaño $240 \times 168 \mathrm{~mm}$. Encuadernado en rústica. Precios: España, 1.200 ptas; extranjero, 17 \$ USA.

\section{SUMARIO:}

Prólogo Prof. G. Ciribini.

\section{Introducción}

Capitulo 1. - La industrialización en las proclamas y manifiestos de arquitectura.

Capítulo 2.-¿Réquiem por la construcción industrializada?

Capitulo 3.-Algunos conceptos básicos. Capítulo 4.- ¿Proyecto tradicional, construcción indus-
trializada?

Capitulo 5.-Componentes.

Capítulo 6. - La coordinación dimensional hoy.

Capítulo 7.-Flexibilidad, intercambiabilidad y catálogos.

Capítulo 8.-Industrialización, normativa y calidad.

Capítulo 9.-Reflexiones finales.

publicación del

INSTITUTO EDUARDO TORROJA 